Article

\title{
Effect of Sediment Concentration on Hydraulic Characteristics of Energy Dissipation in a Falling Turbulent Jet
}

\author{
Wenjuan Gou ${ }^{1}$, Huiping Li ${ }^{1, *}$, Yunyi Du ${ }^{2}$, Hongxia Yin ${ }^{1,3}$, Fang Liu ${ }^{1}$ (I) and Jijian Lian ${ }^{1}$ \\ 1 State Key Laboratory of Hydraulic Engineering Simulation and Safety, Tianjin University, \\ Tianjin 300072, China; gwj@tju.edu.cn (W.G.); 1014205058@tju.edu.cn (H.Y.); fangliu@tju.edu.cn (F.L.); \\ jjlian@tju.edu.cn (J.L.) \\ 2 Environmental Technology Development of TIWTE (Tianjin) Co., Ltd., Tianjin Research Institute for Water \\ Transport Engineering, M.O.T., Tianjin 300072, China; duyunyi1992@hotmail.com \\ 3 School of Water Conservancy and Hydroelectric Power, Heibei University of Engineering, Handan, \\ Hebei 056038, China \\ * Correspondence: lihuiping@tju.edu.cn; Tel.: +86-022-27401137
}

Received: 29 July 2018; Accepted: 13 September 2018; Published: 15 September 2018

Featured Application: This study establishes a foundation that links behavior with sediment concentration to enable further research into energy dissipator function in large dams on sediment-laden rivers.

\begin{abstract}
The effect of sediment on the hydraulics of jet energy dissipation is an urgent issue for high dams built on sediment-laden rivers. Accordingly, flume experiments were conducted using a ski-jump type energy dissipator in flows of four sediment concentrations $\left(0 \mathrm{~kg} / \mathrm{m}^{3}, 50 \mathrm{~kg} / \mathrm{m}^{3}\right.$, $150 \mathrm{~kg} / \mathrm{m}^{3}$, and $250 \mathrm{~kg} / \mathrm{m}^{3}$ ) to determine the effects on discharge, flow regime, and hydrodynamic pressure in a plunge pool. The results demonstrate that the effect of sediment on discharge is constant, regardless of sediment concentration, when compared to fresh water. The width of the nappe decreased with increasing concentrations of sediment. The length of the jet trajectory increased with upstream water head. The time-averaged pressure and fluctuation pressure both exhibited peaks, describing the impact of the jet on the bottom of the plunge pool. The maximum time-averaged pressure and maximum fluctuating pressure both noticeably increased with upstream water head and slightly increased with sediment concentration for a given flow condition. The results also demonstrated that the dominant frequency of fluctuation trends to lower values, and that both the fluctuating energy and vortex scale increase with increasing sediment concentrations due to increased viscosity. These findings can be used to improve energy dissipation in dams on sediment-laden rivers.
\end{abstract}

Keywords: sediment; concentration; energy dissipation; plunge pool; hydraulic characteristics

\section{Introduction}

The interest in energy dissipation in hydraulic structures has developed at an appropriate time, given the significant increase in the construction of large dams worldwide since the 1950s [1]. The design of hydraulic energy dissipators for these dams is often a challenge, especially when dam parameters include high water head and large discharge [2]. The hydraulic jump [3] and ski-jump [4] are common energy dissipation features, as is the plunge pool [5]. However, when a dam is built on a hyperconcentrated sediment-laden river, energy dissipation is complicated by sediment effects [6].

Energy dissipation is an important issue in the design of a hydraulic structure. The hydraulic characteristics of the impinging jet or plunge pool of an energy dissipator have been extensively studied 
in fresh water. Deep outlets with a plunge pool should be considered a standard, common design for energy dissipation [7] and flushing sediment from reservoirs [8]. Nappe flows, in which water is dropped through the atmosphere into a pool or water cushion, exhibit rapid diffusion of the jet in the pool, dissipating the energy of the falling water and attenuating fluctuations at the base of the pool [9]. Many papers have been published on methods to determine the shape and size [10] of a plunge pool and the bottom slab thickness [11], and others have paid special attention to hydraulic plunge pool scour [12]. The safety of plunge pools has also been a subject of close attention, e.g., the hydraulic pressure [13] and uplift force [14]. Other research has concentrated on the mechanism of the impinging jet [15]. The entire flow of an energy dissipator is divided into a free jet region, an impinging region, and wall jet region. Beltaos and Rajaratnam [16] discussed the time-averaged characteristics of an impinging jet and Gutmark et al. [17] presented an experimental study of the turbulent structure on the centerline of a two-dimensional impinging jet. Energy dissipation depends on the turbulence of flow and the fluctuation of turbulent flow based on the formation of coherent structures $[18,19]$. Using experiments and calculations, Adrian et al. [20] concluded that the vortex structure in a wall turbulent flow takes the form of a hairpin. Chakraborty et al. [21] systematically analyzed, summarized, and compared popular vortex identification criteria and vortex structure recognition methods. The scale, intensity, and density of vortex structures have also been studied [22,23]. With the continued development of experimental technology, new advanced simulation methods [24] and devices [25], such as direct numerical simulation (DNS) [26], large eddy simulations (LES) [27], and particle image velocimetry [28], have been used to research turbulence characteristics. However, turbulent flow is a complicated subject, and many details describing turbulent structures still remain inconclusive.

Hyperconcentrated sediment-laden flow has been studied using experiments and simulations over the past century as either a Newtonian fluid [29] or Bingham fluid [30]. The presence and characteristics of particles have been found to affect the turbulent structure of a suspension [31]. Samanta et al. [32] simulated porous turbulent flows using DNS and observed that stream-wise turbulence intensity decreased in a porous duct. The turbulent velocity profile in sediment-laden flows has been studied by experiments [33] and simulations [34,35]. Guo and Julien [36] performed a theoretical analysis and determined that sediment suspension increases main flow energy loss and weakens turbulent diffusion in the vertical direction, and increases the velocity gradient. Wang and Qian [37] performed experiments to study the turbulence structure of open channel flow and confirmed that the turbulence of a sediment-laden flow is less intense, with a smaller frequency and larger turbulence eddies in the longitudinal direction compared with clear water flow; the distribution of density probability and the autocorrelation coefficients of the fluctuating velocity were similar to that of a clear water flow. These results suggested that the fundamental turbulence structure of a sediment-laden Newtonian flow remains unchanged, but Wang et al. [38] found different experimental results. Omid et al. [39] and Nasrabadi et al. [40] performed experiments demonstrating that suspended sediment increases flow resistance as a result of decreased maximum flow velocity. Though a great deal of the literature published in the last century has addressed the transport of sediment and turbulent structure of sediment-laden flow using experiments [41] and simulations [42], it remains necessary to clarify the effects of sediment-laden flow on ski-jump type energy dissipators, as they appear to complicate the turbulence structure and energy dissipation mechanisms of a fluid.

For a high dam in a hyperconcentrated, sediment-laden river, an impinging jet from a deep outlet and plunge pool are combined to act as an energy dissipator. Deep outlets perform the critical function of flushing sediment from behind a dam, so the hydraulics of hyperconcentrated flows should be determined in order to ensure safe operation. The effect of sediment on energy dissipation is an urgent issue for dams on hyperconcentrated sediment-laden rivers. In this paper, experiments were conducted using a re-circulating flume and deep outlet discharge subjected to flow of four sediment concentrations $\left(0 \mathrm{~kg} / \mathrm{m}^{3}, 50 \mathrm{~kg} / \mathrm{m}^{3}, 150 \mathrm{~kg} / \mathrm{m}^{3}\right.$, and $\left.250 \mathrm{~kg} / \mathrm{m}^{3}\right)$ and three upstream water heads $(1.8 \mathrm{~m}, 1.85 \mathrm{~m}, 1.9 \mathrm{~m})$ to study the effects of sediment concentration on the hydraulics of energy 
dissipation, as quantified by discharge, flow regime of nappes, and hydraulic pressure on the slab in the plunge pool.

\section{Experimental Setup}

The experiments were conducted using a re-circulating flume, shown schematically in Figure 1a. The flume mainly consisted of an upstream tank with a water level indicator supporting a stabilized water head, a plunge pool, and a pump with a flow meter. The upstream tank was $1 \mathrm{~m}$ wide, $1.3 \mathrm{~m}$ long and $2.6 \mathrm{~m}$ high. The $0.61 \mathrm{~m}$ deep plunge pool was $4 \mathrm{~m}$ long and of a trapezoidal cross-section, $0.8 \mathrm{~m}$ and $0.4 \mathrm{~m}$ wide at the top and bottom, respectively. A deep outlet was fixed at the upstream tank $1.28 \mathrm{~m}$ above the bottom plate of the plunge pool, as shown in the schematic diagram in Figure $1 \mathrm{~b}$. The tank and the plunge pool were connected by $12 \mathrm{~cm}$ diameter pipes and a mud pump.

The tests were conducted in a room-temperature environment of about $20 \pm 2{ }^{\circ} \mathrm{C}$. Upstream and downstream water levels were controlled within an experimental error of $\pm 1 \mathrm{~mm}$. The flow discharge was measured by a flow meter, and each experiment case was conducted three times to determine the average discharge. Diaphragm-type TS202 pressure transducers (Beijing Tiantai Xingye Technology co. Ltd, Beijing, China)with a diameter of $20 \mathrm{~mm}$ were spaced at $0.1 \mathrm{~m}$ intervals along the centerline of the plunge pool to measure the hydrodynamic pressure. A DASP data collection system sampled the transducers at $100 \mathrm{~Hz}$ on the basis of Nyquist's law [43], equivalent to a sampling time interval of $0.01 \mathrm{~s}$. The transducers were calibrated in the air and the hydrostatic pressures were measured prior to the experiments in order to provide a comparison of water level before and after the experimental hydrodynamic pressures were applied. At least 8192 effective samples were taken in every experimental case. The complete set of samples was first analyzed for effective data, then decomposed using an infinite impulse response (IIR) filter in MATLAB (The MathWorks, Inc., Natick, MA, U.S.). Finally, the parameters of the hydrodynamic pressures were calculated using MATLAB.

The sediment used was from the Yellow River and was classified as fine silt and clay of particle sizes ranging from $0.001 \mathrm{~mm}$ to $0.05 \mathrm{~mm}$ and a median particle size of $0.008 \mathrm{~mm}$. The concentration of mud was determined by its concentration at the nappe. Viscosity was measured by an NDJ-8S (Shanghai Yueping Scientific Instrument Co. Ltd., Shanghai, China) rotational viscometer. After testing each case, the viscosity of the sediment-laden flow was measured at room temperature. Each case was repeated five times and the results were averaged for analysis.

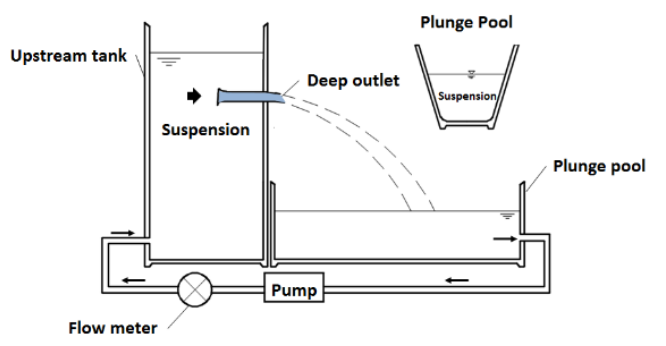

(a)

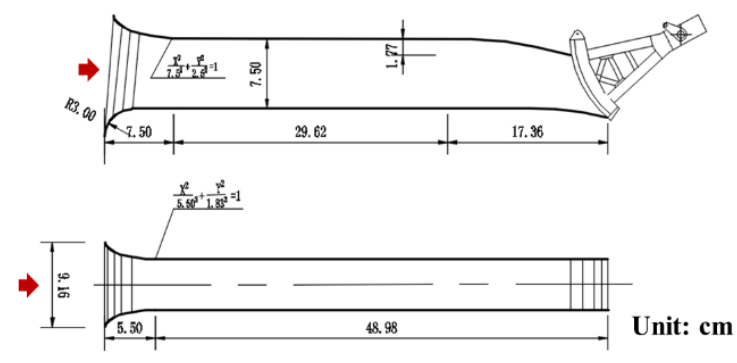

(b)

Figure 1. Schematic diagram of the experimental (a) re-circulating flume; and (b) deep outlet.

Experiments were conducted in two sets for a downstream water depth of $0.1 \mathrm{~m}$. The first set of experiments evaluated fresh water at three upstream water heads $(1.80 \mathrm{~m}, 1.85 \mathrm{~m}$, and $1.90 \mathrm{~m})$ to determine its energy dissipation in the plunge pool. The second set of experiments consisted of three concentrations $\left(50 \mathrm{~kg} / \mathrm{m}^{3}, 150 \mathrm{~kg} / \mathrm{m}^{3}\right.$, and $\left.250 \mathrm{~kg} / \mathrm{m}^{3}\right)$ of sediment-laden flow to determine its energy dissipation at each of the three evaluated upstream water heads. The densities of the suspensions were $1.02 \mathrm{~kg} / \mathrm{m}^{3}, 1.05 \mathrm{~kg} / \mathrm{m}^{3}$, and $1.08 \mathrm{~kg} / \mathrm{m}^{3}$ for sediment concentrations of $50 \mathrm{~kg} / \mathrm{m}^{3}, 150 \mathrm{~kg} / \mathrm{m}^{3}$, and $250 \mathrm{~kg} / \mathrm{m}^{3}$, respectively. The details of all twelve experimental cases are presented in Table 1 . The experimental errors were controlled to less than $1 \%$. 
Table 1. Experimental cases.

\begin{tabular}{cccccccc}
\hline No. & $\begin{array}{c}\text { Concentrations } \\
\text { of Sediment } \\
\left(\mathbf{k g} / \mathbf{m}^{\mathbf{3}}\right)\end{array}$ & $\begin{array}{c}\text { Upstream } \\
\text { Water } \\
\text { Head }(\mathbf{m})\end{array}$ & $\begin{array}{c}\text { Downstream } \\
\text { Water } \\
\text { Depth }(\mathbf{m})\end{array}$ & No. & $\begin{array}{c}\text { Concentrations } \\
\text { of Sediment } \\
\left(\mathbf{k g} / \mathbf{m}^{\mathbf{3}}\right)\end{array}$ & $\begin{array}{c}\text { Upstream } \\
\text { Water } \\
\text { Head }(\mathbf{m})\end{array}$ & $\begin{array}{c}\text { Downstream } \\
\text { Water Depth } \\
(\mathbf{m})\end{array}$ \\
\hline 1 & 0 & 1.80 & 0.1 & 7 & 150 & 1.80 & 0.1 \\
2 & 0 & 1.85 & 0.1 & 8 & 150 & 1.85 & 0.1 \\
3 & 0 & 1.90 & 0.1 & 9 & 150 & 1.90 & 0.1 \\
4 & 50 & 1.80 & 0.1 & 10 & 250 & 1.80 & 0.1 \\
5 & 50 & 1.85 & 0.1 & 11 & 250 & 1.85 & 0.1 \\
6 & 50 & 1.90 & 0.1 & 12 & 250 & 1.90 & 0.1 \\
\hline
\end{tabular}

\section{Experimental Results and Analysis}

\subsection{Flow Discharge}

Flow discharge is a critical control factor in the design of a hydraulic structure. A satisfactory flow rate ensures the safe operation of a dam in a hyperconcentrated sediment-laden river, so the effect of sediment on flow discharge is an important and urgent issue. The experiments in this study were conducted to determine the relationship between flow rate and upstream water head for sediment-laden flow. The experimental results are presented in Figure 2, in which the dark line is the flow rate of fresh water and the dots are the flow rates of the sediment-laden flow of various sediment concentrations. The flow rate was observed to increase exponentially with the square root of the upstream water head. The dots were close to the line, indicating that the flow rate is stable regardless of sediment concentration, which is the same conclusion reached by references [44,45]. Therefore, when a hydraulic structure is designed, the flow rate need only be evaluated in terms of fresh water.

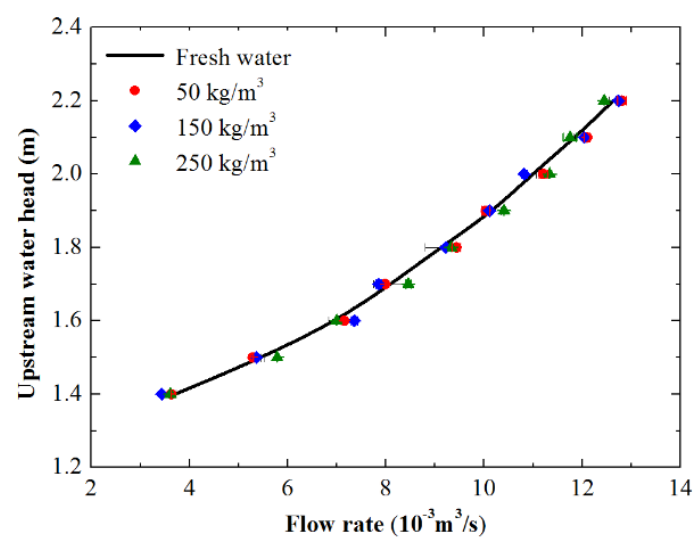

Figure 2. Relationship between flow rates and upstream water head to deviations at different sediment concentrations.

\subsection{Jet Properties}

Jet properties affect the mean or fluctuating pressure on the floor slab of a plunge pool because the break-up and air entrainment of the jet is influenced by surface tension and turbulence effects. These effects, in turn, determine the thickness of the slab. Nappe regimes at various sediment concentrations are shown in Figure 3. The air entrainment of the falling jet is obvious. The break-up of the jet in the air is best understood by vertically dividing it into three major flow regimes. The first region was characterized by the initial formation of waves on the surface of the jet. Surface tension resists the growth of these disturbances. The flow exiting the outlet was glassy and smooth, and the nappe section was similar to that of a sharp outlet. This glassy smooth region increased in length with increasing sediment concentrations. The second region was characterized by the growth of instabilities on the water surface. Small, regularly spaced waves formed and were amplified in the direction of flow. Notably, the falling jet of sediment-laden flow was smoother than that of fresh water, and the 
small waves appeared slightly later. The third region was characterized by the transition of the surface waves into circumferential vortex elements. Jet break-up was clearly observed and was less intense in sediment-laden flow than in fresh water, as was the air entrainment. This behavior has been attributed to the higher viscosity of sediment-laden flow [46]. Figure 4 presents the relationship between viscosity and sediment concentration at $10{ }^{\circ} \mathrm{C}, 20^{\circ} \mathrm{C}$, and $30^{\circ} \mathrm{C}$. Clearly, the viscosity of flow increases with increasing sediment concentration and decreases with increasing temperature. The increasing viscosity of the sediment-laden flow likely results in an increase in surface tension [9], and, as a result, the falling jets exhibit different nappe regimes at the different sediment concentrations.

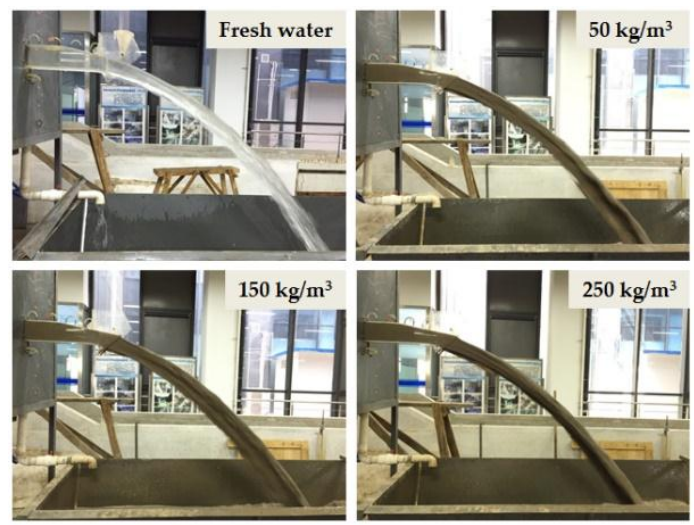

Figure 3. Nappe regimes at different sediments concentrations.

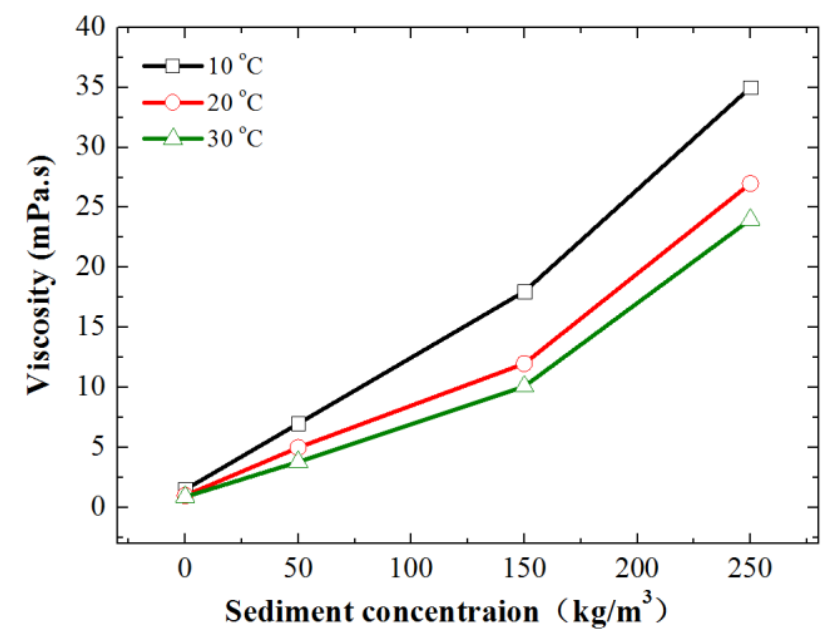

Figure 4. Relationship between various sediment concentrations and fluid viscosity.

When a falling jet impacts the floor slab of a plunge pool of sufficient depth, the flow regime in the plunge pool can be divided into the three regions shown in Figure 5 to determine the hydraulic pressure distribution. The first region (I) is the submerged jet region. In this region, the flow velocity of the main stream remains approximately linear; the center of the main stream exhibits the highest velocity while the edge of the main stream exhibits the lowest velocity. The second region (II) is the main stream impact region, in which the velocity of the main stream decreases rapidly and streamlines abruptly upon meeting the slab, so the pressure on the slab of the plunge pool increases sharply. The third region (III) is the wall jet region, in which the main stream is ejected and flows to the wall and along the slab of the plunge pool. Here, spirals begin to roll and diffuse. Looking at these three regions, clearly the design impact pressure is caused by the effects of the jet in the main stream impact region (II), where the maximum pressure on the slab occurs, and instability and failure of the slab are most likely. 


\subsection{Hydrodynamic Pressure}

Hydrodynamic pressure was measured along the centerline of the plunge pool by diaphragm-type TS202 pressure transducers and a DASP data collection system. According to the results of previous research [44], a sampling frequency of $100 \mathrm{~Hz}$ was used that satisfied Nyquist's law [43]. The selection of sample interval and sampling time is related to the ability of the collected data to accurately reflect the reality of the experiment. According to the sampling theorem, the truncation frequency of Nyquist's law must be greater than the highest frequency of the sampling signal in the selection. If the sampling interval is too large, the truncation frequency is too small, causing spectrum aliasing. As a result, at least 8192 samples were taken for every experiment in order to provide sufficient data to reduce uncertainty [25]. Figure 6 depicts a portion of the original time history curve of hydrodynamic pressures for Case 3. These data were then decomposed by an IIR filter before proceeding with the analysis.

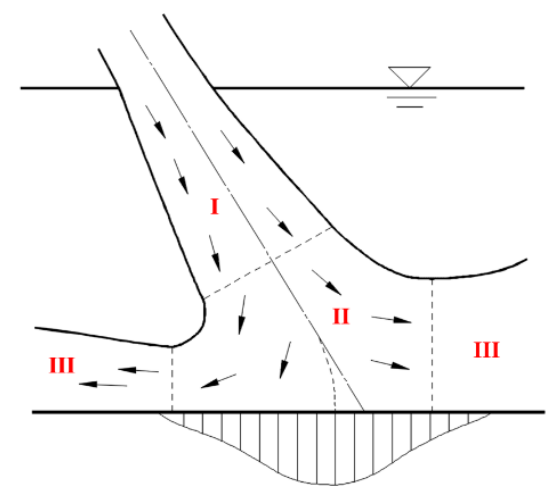

Figure 5. Schematic diagram of the flow regime.

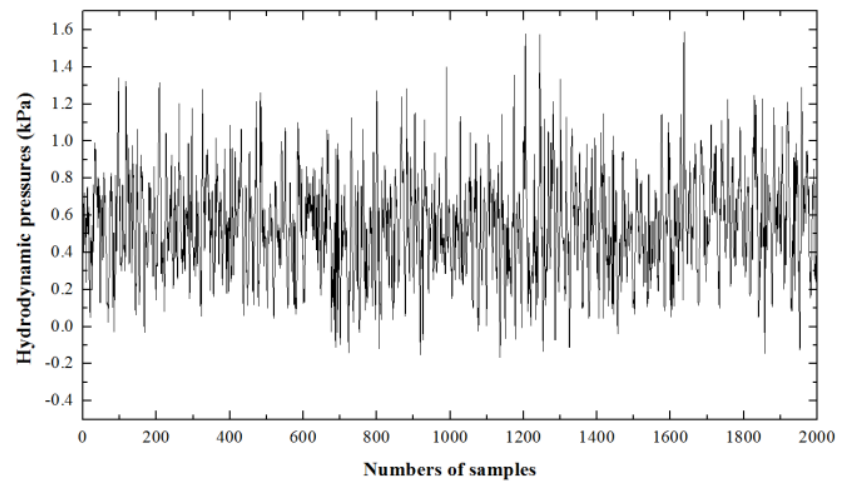

Figure 6. The time history curve of maximum hydrodynamic pressures for Case 3.

Hydrodynamic pressure can be expressed as a combination of mean pressure and fluctuating pressure as

$$
P_{i}=\bar{P}+P^{\prime}
$$

where $P_{i}$ is the instantaneous pressure at a test point, $\bar{P}$ is the mean pressure at this test point, and $P^{\prime}$ is the fluctuating pressure. Mean and fluctuating dynamic pressure on the centerline of the floor slab is affected by the diffusion and air entrainment in the falling jet, and the scale of the turbulence in the plunge pool. 


\subsubsection{Mean Pressure}

The mean dynamic pressure of the falling jet on the plunge pool floor slab can be determined by the average of the dynamic pressure over time, thus, the relative time average pressures $\left(P^{*}\right)$ can be given as

$$
P^{*}=\bar{P}-h_{0}
$$

where $\bar{P}$ is the time-averaged pressure at every test point and $h_{0}$ is the static water depth in the plunge pool. The relative time-averaged pressure is a function of the location of the experimental measurement points, as presented in Figure 7. The experimental results illustrate that the relative time-averaged pressures remain stable before exhibiting a clear peak, as can be observed in Figure 7a-c. This peak in relative pressure mainly occurs in the main stream impact region, which is clearly similar to the experimental results of $\mathrm{Xu}$ et al. [5]. In this region, the velocity of the jet is forced to sharply decrease, causing the pressure to abruptly increase [9]. The maximum value of the relative pressure, which affects the stability and safety of the plunge pool slab [13], can be seen in Figure 7 to increase with increasing sediment concentration, agreeing well with the experimental results of Wang and Qian [37]. This result was expected, because the higher pressure can be explained by the increase in the viscosity of the liquid that accompanies increased sediment concentration, causing the diffusion and air entrainment of the jet to decrease.

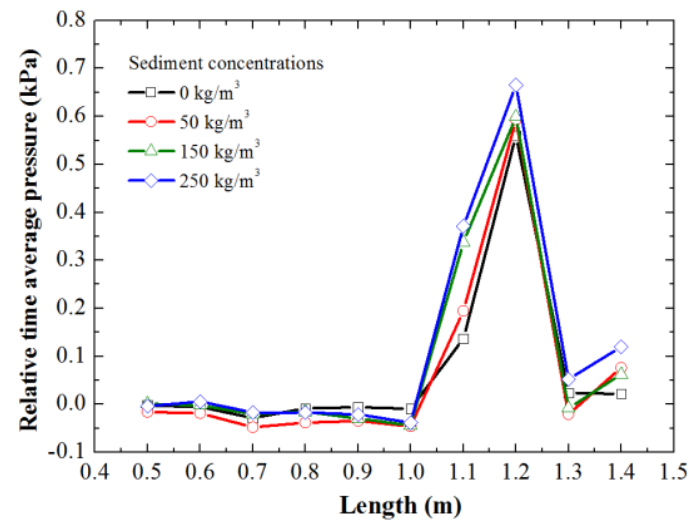

(a)

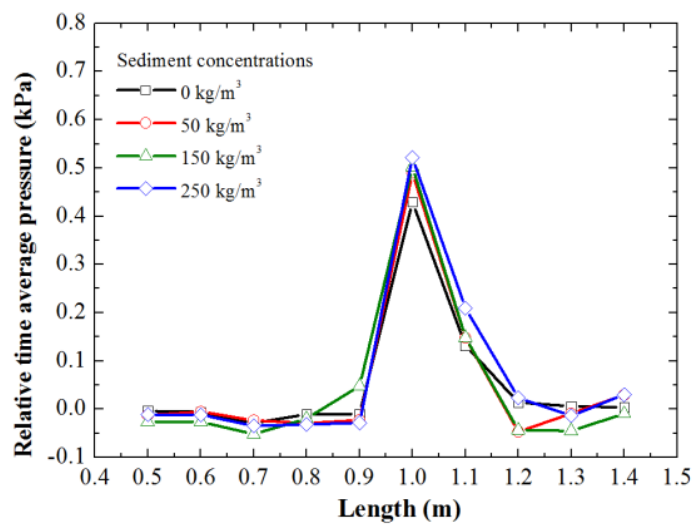

(c)

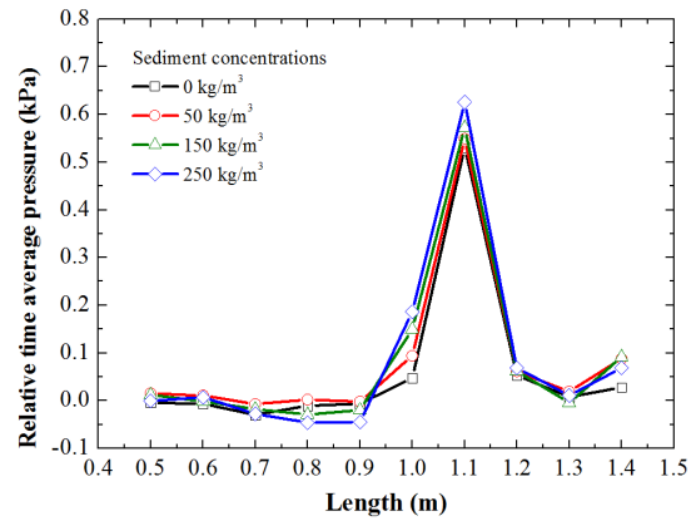

(b)

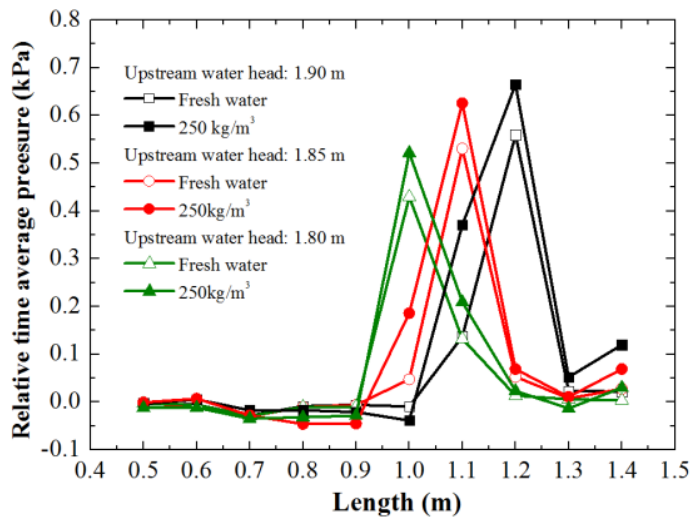

(d)

Figure 7. Time-averaged pressure and location for different upstream water heads at different concentrations of sediments: (a) Upstream water head of $1.9 \mathrm{~m}$; (b) upstream water head of $1.85 \mathrm{~m}$; (c) upstream water head of $1.8 \mathrm{~m}$; (d) comparison of pressures at different upstream water heads due to fresh water and sediment-laden flow of $250 \mathrm{~kg} / \mathrm{m}^{3}$ in concentration.

With increasing upstream water head, the pressure peak moves downstream, as shown in Figure $7 \mathrm{~d}$, indicating that the impact region of the jet moves downstream as it travels further 
horizontally through the air. The difference in the maximum values of relative pressure caused by fresh water and by water with a concentration of $250 \mathrm{~kg} / \mathrm{m}^{3}$ is approximately stable, regardless of upstream water head. It is likely that the increase in upstream water head has little influence on the comparative degree of turbulence of fresh water and sediment-laden water. Clearly, while pressure head does not change the relative effects of sediment-laden flow, the sediment concentration has an obvious influence on the impact pressure.

\subsubsection{Fluctuating Pressure}

The mean hydrodynamic pressure represents the average falling jet force on the floor slab of the plunge pool over a period of time, and the fluctuating pressure caused by turbulence represents the degree of turbulence at every point tested. The pressure fluctuation on the center line of the plunge pool was measured by transducers and the root mean squares (RMS) of the fluctuating pressure were determined by

$$
\sigma=\sqrt{\frac{1}{N} \sum_{i=1}^{N}\left(P_{i}-\bar{P}\right)^{2}}
$$

where,

$$
\bar{P}=\frac{1}{N} \sum_{i=1}^{N} P_{i}
$$

in which $\sigma$ is the RMS fluctuating pressure, $P_{i}$ is the instantaneous pressure at a given point, $i$ is the test number, $\bar{P}$ is the mean value of pressure at all test points, and $N$ is total number of tests. As shown in Figure $8 \mathrm{a}-\mathrm{c}$, the experimental results indicate that the RMS fluctuating pressures remain stable at the beginning of the plunge pool, then increase sharply, exhibiting a peak before returning to a stable value at the end. For sediment-laden flow, the maximum values of the RMS pressure increased with increasing sediment concentration from $0 \mathrm{~kg} / \mathrm{m}^{3}$ to $250 \mathrm{~kg} / \mathrm{m}^{3}$ for a given upstream water head. The increasing concentration of sediment causes an increase in the viscosity of the flow, resulting in a decreased turbulence intensity [35] and an increased fluctuation in turbulence, which is consistent with the turbulence characteristics of sediment-laden flows in open channels [34].

Figure $8 \mathrm{~d}$ shows that the maximum RMS increased with increasing upstream water head at a given concentration of sediment, and shows that the location of the maximum RMS moved downstream with the length of the region following the peak increasing with increasing water head. This indicates that the higher the upstream water head, the more energy carried by the jet and then applied to the slab, and the larger "hill" region following the peak of the RMS indicates that the main stream impact region is larger. Figure 8d, in which same color lines represent the RMS measured for different sediment concentrations at the same upstream water head, further demonstrates this relationship: The differences between the maximum RMS at different concentrations are similar, regardless of the upstream water head.

Figure 9 depicts the maximum time-averaged pressure and the maximum fluctuating pressure for different sediment concentrations at different upstream water heads. The maximum time-averaged pressure can be seen to increase with both sediment concentration and water head, but the slopes at which the pressure increases with sediment concentration for all three water heads are nearly identical. The same trends are exhibited for the maximum fluctuating pressure. The results demonstrate that the pressure increases in a stable manner with the increasing sediment concentrations regardless of the upstream water head owing to the viscosity of the suspension and the accompanying flow resistance. Nasrabadi et al. [40] conducted experiments studying a submerged hydraulic jump with sediment-laden flow and determined that suspended sediment increased the flow resistance as a result of the decreased maximum flow velocity, which likely further confirms the presently observed effects of sediment in the flow. 


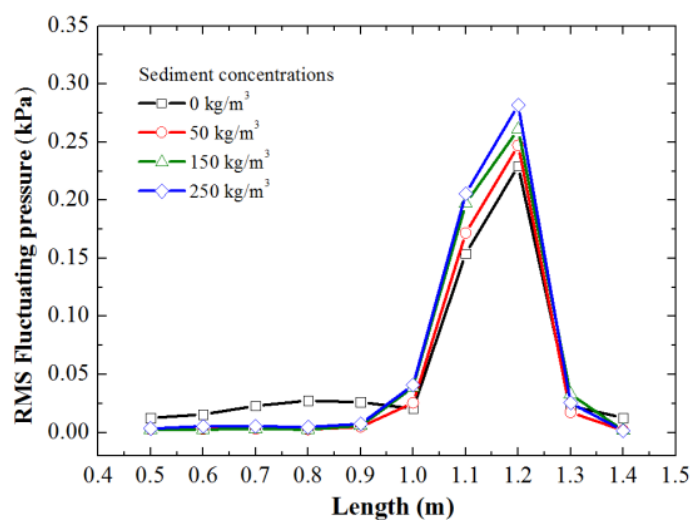

(a)

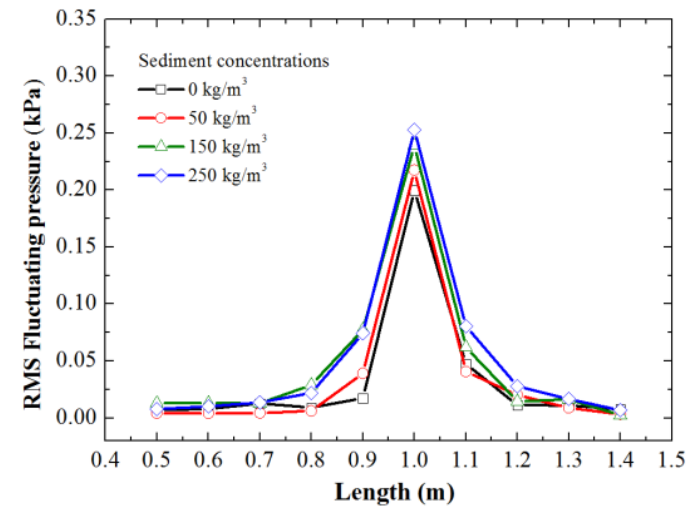

(c)

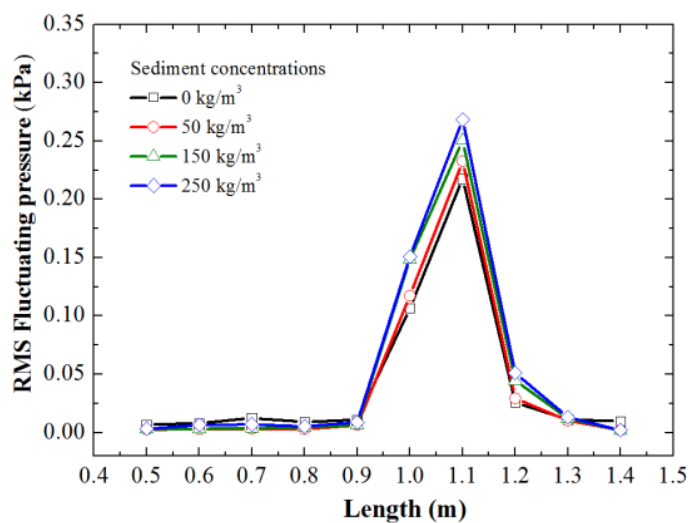

(b)

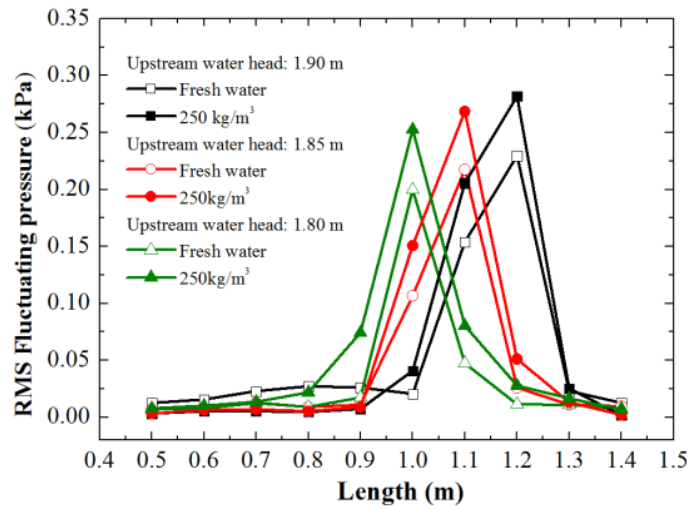

(d)

Figure 8. Relationship between fluctuating pressure and upstream water head for different concentrations of sediments: (a) Upstream water head of $1.90 \mathrm{~m}$; (b) upstream water head of $1.85 \mathrm{~m}$; (c) upstream water head of $1.80 \mathrm{~m}$; (d) comparison of pressures measured at different upstream water heads for fresh water and sediment-laden flow of $250 \mathrm{~kg} / \mathrm{m}^{3}$ in concentration.

\subsubsection{Amplitude Characteristics}

The maximum fluctuating pressure is further studied here as a key characteristic of jet flow. The probability density is an important statistical parameter describing fluctuating pressure and representing its overall amplitude. When the upstream water head was $1.90 \mathrm{~m}$, the maximum pressure on the slab of the plunge pool was $1.20 \mathrm{~m}$ from the wall of the upstream tank. Figure 10 depicts the probability density of the maximum fluctuating pressure for different concentrations of sediments at an upstream water head of $1.90 \mathrm{~m}$. In this figure, the probability densities of the maximum pressure at different concentrations basically satisfy a Gaussian distribution and are positively skewed, i.e., the positive fluctuations tend to be larger than the negative ones. The peaks of the probability densities decrease, and the weights of the probability densities increase, with increasing sediment concentration. Wang and Qian [37] conducted experiments studying the turbulence structure of open channel flow and suggested that the turbulence in sediment-laden flow is less intense than that in clear water flow. This further demonstrates that the turbulence intensity decreases, and the fluctuation of turbulence increases, with increasing sediment concentration. 


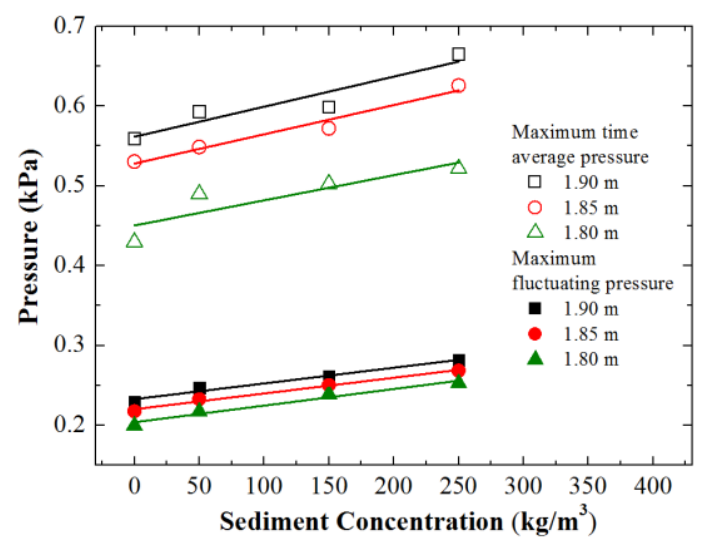

Figure 9. Relationship between pressures and sediment concentrations for different upstream water heads.

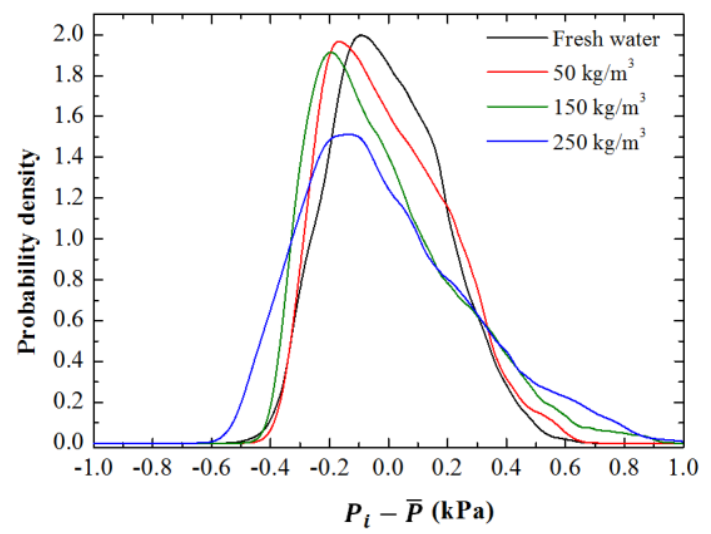

Figure 10. Probability densities for peak pressure at $1.20 \mathrm{~m}$ from the upstream tank at different sediment concentrations and an upstream water head of $1.90 \mathrm{~m}$.

\subsubsection{Frequency Domain Characteristics}

The power spectrum is an important parameter that represents the frequency domain characteristics of fluctuating pressure, given by

$$
S(f)=4 \int_{0}^{\infty} R(t) \cos 2 \pi f t d t
$$

where $f$ is the frequency of fluctuating pressure, $t$ is the test time, and $S(f)$ is the power spectrum of the fluctuating pressure. The maximum fluctuating pressure on the slab of the plunge pool is also a key characteristic for studying the frequency domain characteristics. Figure 11 depicts the relationships of the power spectrum and frequency for an upstream water head of $1.90 \mathrm{~m}$ at different sediment concentrations. The calculations show that the turbulence energy was most concentrated at frequencies of $0-0.2 \mathrm{~Hz}$ and that the frequency bands of the fluctuating energy widen with increasing sediment concentrations. The dominant frequency trended to a lower frequency with increasing sediment concentration. The results demonstrate that the higher the sediment concentration, the larger the viscosity of the flow and the lower the turbulent kinetic energy of the small-scale vortexes, which decrease gradually, causing the turbulent kinetic energy to become more concentrated in large-scale energetic vortexes. Notably, under these conditions, the turbulence eddies are longer. These results are similar to the results of Wang and Qian [37] who undertook experiments to study turbulent structure of open channel flow with plastic particles. Figure 12 depicts the relationships of spectral density and frequency for four concentrations $(0 \%, 1.4 \%, 5.6 \%$ and $10.4 \%)$ of particles with a mean diameter of $0.266 \mathrm{~mm}$. They reveal that the turbulent frequency decreases with the increase 
in concentration and the turbulence energy is transmitted to the eddy with low frequency and large size. However, the different values of frequencies by the two experiments are the result of different turbulence and flow regimes.

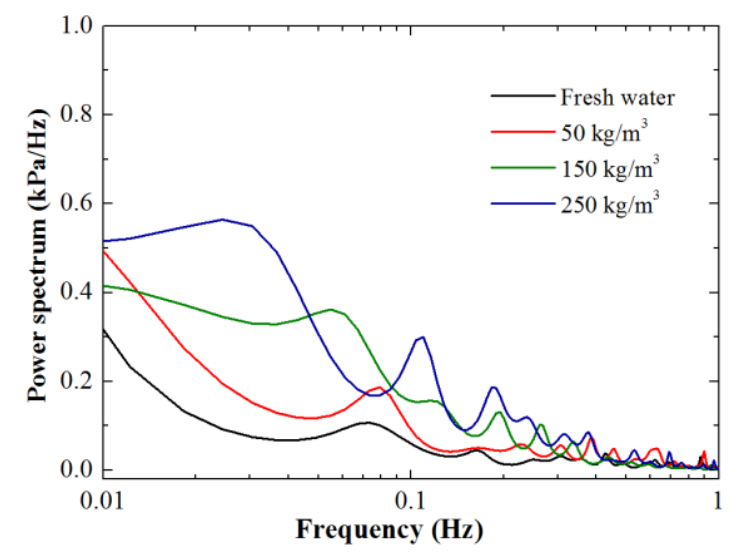

Figure 11. Relationships between power spectrum and frequency with an upstream water head of $1.90 \mathrm{~m}$ for different sediment concentrations.

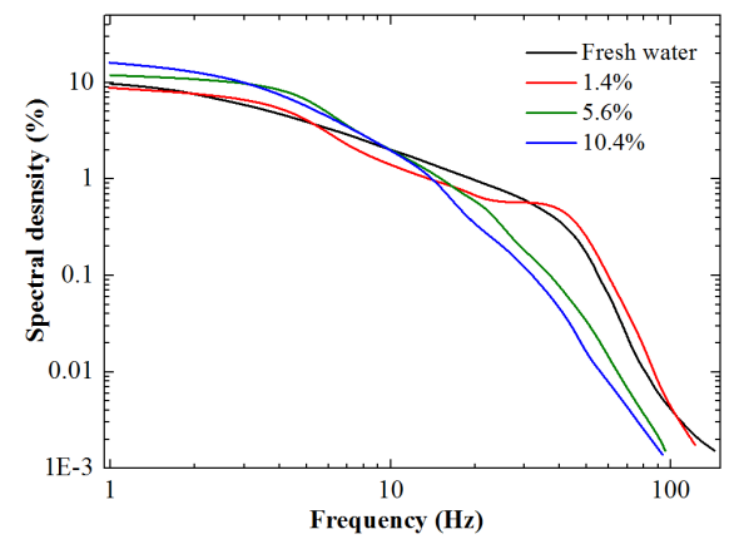

Figure 12. Relationships of spectral density distribution with concentrations taken by Wang and Qian [37].

\subsubsection{Time Domain Characteristics}

The coefficient of association is an important parameter used to analyze the turbulent structure and dimensions of vortexes. The coefficient of association includes two parameters: The coefficient of autocorrelation and the coefficient of cross-correlation. The point of maximum pressure is the critical location at which the coefficient of association, $R$, should be determined, calculated by

$$
R=\frac{1}{T-\tau} \int_{0}^{T-\tau}\left[p_{x}(t)-\overline{p_{x}}\right] \cdot\left[p_{y}(t+\tau)-\overline{p_{y}}\right] d t
$$

where $T$ is the entire test duration; $t$ is the test time and $\tau$ is an interval of time; $p_{x}$ is the hydrodynamic pressure at a given test point and $p_{y}$ is the hydrodynamic pressure at another test point; $\overline{p_{x}}$ and $\overline{p_{y}}$ are the average hydrodynamic pressures at test points $x$ and $y$, respectively; when $p_{x}$ and $p_{y}$ represent a single test point, $R$ represents the coefficient of auto correlation for $R_{x x}$, and when $p_{x}$ and $p_{y}$ represent two different test points, $R$ represents the coefficient of cross-correlation for $R_{x y}$.

The coefficient of autocorrelation of the maximum hydrodynamic pressure, and the coefficient of cross-correlation between the point with the maximum pressure and the other points located at $0.1 \mathrm{~m}$ intervals downstream, should be calculated. By comparing the coefficients of autocorrelation of the fresh water and water with a sediment concentration of $250 \mathrm{~kg} / \mathrm{m}^{3}$, the results in Figure 13 demonstrate 
that the eddy sizes in sediment-laden flow are larger than those in fresh water. Additionally, the coefficients of cross-correlation of the fresh water and water with a sediment concentration of $250 \mathrm{~kg} / \mathrm{m}^{3}$ indicate that the scale of the vortex increases with the presence of sediment in the flow. The scale of the vortex increases when sediment is present as a result of the changes in turbulent structure and surface tension $[47,48]$.

Although different objects were studied, and different experimental details were produced, these results correspond well with those in reference [37], as shown in Figure 14. Figure 14, taken from Wang and Qian [37] depicts the relationships of eddy sizes with particle concentrations. The results reveal that the longitudinal sizes of the macroscale eddies increase with the increase in sediment concentration and is mainly decided by the low frequency, large-size eddies. When the frequency of turbulent eddies is reduced, the energy proportion carried by large-size eddies increases. The longitudinal sizes of the microscale eddies increase with an increase in concentration and is mainly decided by small-size eddies with high frequency. For a sediment-laden flow, part of the energy carried by high frequency eddies decreases and the smallest eddies vanish.

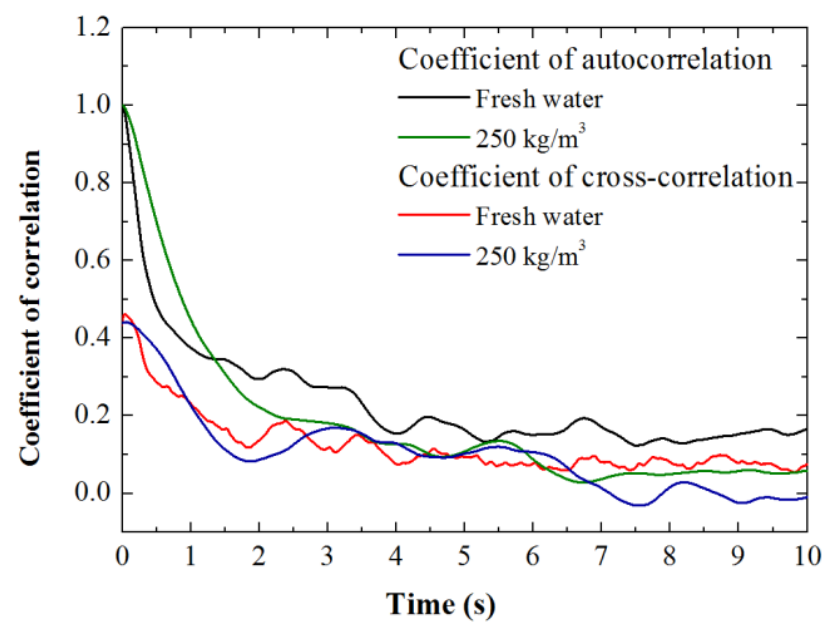

Figure 13. Relationships between coefficient of association at peak pressure and time for fresh water and sediment-laden flow of $250 \mathrm{~kg} / \mathrm{m}^{3}$ concentration at an upstream water head of $1.90 \mathrm{~m}$.

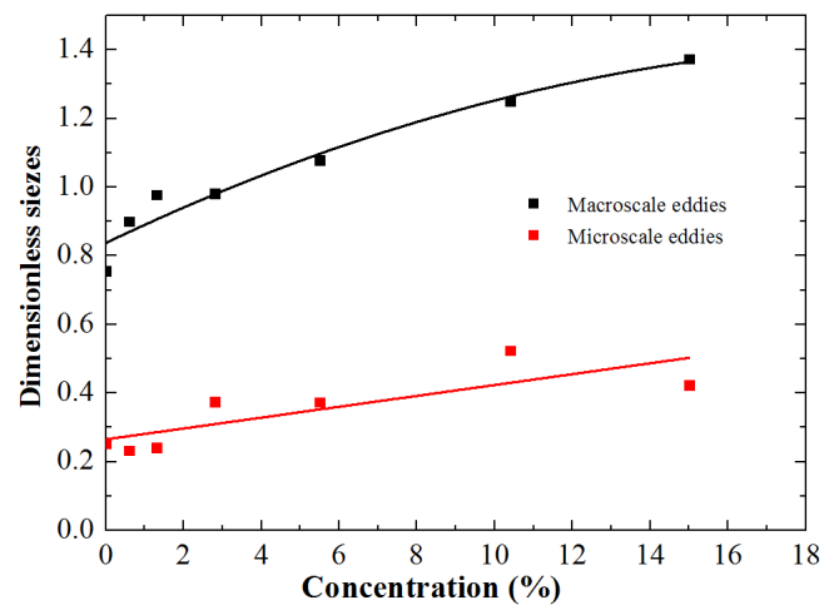

Figure 14. Relationships between eddy sizes and particle concentrations taken by Wang and Qian [37].

Hence, the fluctuating pressure is the result of the movement and interaction of different scales of vortexes in the plunge pool, caused by the nappe jet. The results of the experiments and calculations demonstrate that the larger the sediment concentration in the liquid of the jet, the higher its viscosity. This causes a decrease in vortexes of smaller scales and an increase in vortexes of larger scales, leading to an increase in fluctuating pressure and, thus, energy. The scale of vortexes increases with the 
increasing concentrations of sediments. The energy of fluctuation also increases with increasing concentrations of sediments.

\section{Conclusions}

The effect of the concentration of fine sediments on the hydraulics of the energy dissipation of a turbulent jet is an important and urgent problem for dams on hyperconcentrated sediment-laden rivers. Experiments were conducted using a deep outlet in an upstream tank and a plunge pool consisting of 12 test cases at four sediment concentrations $\left(0 \mathrm{~kg} / \mathrm{m}^{3}, 50 \mathrm{~kg} / \mathrm{m}^{3}, 150 \mathrm{~kg} / \mathrm{m}^{3}\right.$ and $\left.250 \mathrm{~kg} / \mathrm{m}^{3}\right)$ under three upstream water heads $(1.80 \mathrm{~m}, 1.85 \mathrm{~m}$ and $1.90 \mathrm{~m})$. The results of these tests were used to study the hydraulic characteristics of energy dissipation in terms of flow discharge, jet properties, and hydrodynamic pressure. The main conclusions are:

(1) Flow discharge is solely related to the upstream water head for a deep outlet and is independent of sediment concentration.

(2) The horizontal length of the jet from the deep outlet to the plunge pool increases with increasing upstream water head, and the width of the jet decreases with increasing sediment concentrations. The nappe of the jet also becomes smoother and exhibits decreased air entrainment as a result of the higher viscosity of suspensions with higher sediment concentrations.

(3) The time-averaged pressure in the plunge pool exhibits a peak around the impact region of the falling jet and the magnitude and distance from the outlet of this peak increases with increasing sediment concentration due to increased viscosity. The distance from the outlet to the peak also increases with increasing upstream water head.

(4) The RMS fluctuating pressure exhibits the same trend as the time-averaged pressure. The probability density, power spectrum, and coefficient of correlation of hydrodynamic pressure was calculated and the results demonstrate that as sediment concentration increases, the fluctuating energy increases, as does the scale of the vortexes, and the dominant frequency decreases.

The findings of this study provide invaluable insights into the effects of sediment on the behavior of energy dissipators in large river dams. The behaviors and relationships determined in this study establish a foundation for the informed design of dam energy dissipator structures such as plunge pools, improving the operational safety of the overall dam. In the future, the effects of sediment on the velocity profile of the flow will be studied further.

Author Contributions: All authors contributed to the research work. J.L., H.L. and W.G. conceived and designed the experiments; Y.D., H.Y. and F.L. performed the tests; H.L. analyzed the data; and W.G. drafted the paper.

Funding: The authors are grateful to the National Natural Science Foundation of China (Grant Nos. 51509180, 51579172 and 51409187) for financial support.

Acknowledgments: The authors dedicate the manuscript to the memory of Min Yang who gave much advice.

Conflicts of Interest: The authors declare no conflict of interest.

\section{References}

1. Chanson, H. Energy Dissipation in Hydraulic Structures; IAHR Monograph; CRC Press, Taylor \& Francis Group: Leiden, The Netherlands, 2015; p. 168. ISBN 978-1138027558.

2. Lian, J.; Qi, C.; Liu, F.; Gou, W.; Pan, S.; Ouyang, Q. Air entrainment and air demand in the spillway tunnel at the Jinping-I dam. Appl. Sci. 2017, 7, 930. [CrossRef]

3. Hager, W.H.; Bremen, R. Classical hydraulic jump-sequent depths. J. Hydraul. Res. 1989, $27,565-585$. [CrossRef]

4. Heller, V.; Hager, W.H.; Minor, H.E. Ski jump hydraulics. J. Hydraul. Eng. 2005, 131, 347-355. [CrossRef]

5. Xu, W.; Liao, H.; Yang, Y.; Wu, C. Turbulent flow and energy dissipation in plunge pool of high arch dam. J. Hydraul. Res. 2002, 40, 471-476. [CrossRef] 
6. Shu, A.; Liu, Q.; Yi, Y.; Zhang, Z. Characteristics of energy dissipation in hyperconcentrated flows. Int. J. Sediment Res. 2008, 23, 387-397. [CrossRef]

7. Lian, J.; Liu, X.; Ma, B. Safety evaluation and the static-dynamic coupling analysis of counter-arched slab in plunge pool. Sci. China Ser. E Technol. Sci. 2009, 52, 1397-1412. [CrossRef]

8. Shen, H.W. Flushing sediment through reservoirs. J. Hydraul. Res. 1999, 37, 743-757. [CrossRef]

9. Ervine, D.A.; Falvey, H.T.; Withers, W. Pressure fluctuations on plunge pool floors. J. Hydraul. Res. 1997, 35, 257-279. [CrossRef]

10. Manso, P.F.A.; Bollaert, E.F.R.; Schleiss, A.J. Evaluation of high-velocity plunging jet-issuing characteristics as a basis for plunge pool analysis. J. Hydraul. Res. 2008, 46, 147-157. [CrossRef]

11. Manso, P.A.; Bollaert, E.F.R.; Schleiss, A.J. Influence of plunge pool geometry on high-velocity jet impact pressures and pressure propagation inside fissured rock media. J. Hydraul. Eng. 2009, 135, 783-792. [CrossRef]

12. Pagliara, S.; Hager, W.H.; Minor, H.E. Hydraulics of plane plunge pool scour. J. Hydraul. Eng. 2006, 132, 450-461. [CrossRef]

13. Puertas, J.; Dolz, J. Plunge pool pressure due to a falling rectangular jet. J. Hydraul. Eng. 2005, 131, $404-407$. [CrossRef]

14. Melo, J.F.; Pinheiro, A.N.; Ramos, C.M. Forces on plunge pool slabs: Influence of joints location and width. J. Hydraul. Eng. 2006, 132, 49-60. [CrossRef]

15. Qian, S.; Wu, J.; Ma, F. Hydraulic performance of ski-jump-step energy dissipater. J. Hydraul. Eng. 2016, 142, 05016004. [CrossRef]

16. Beltaos, S.; Rajaratnam, N. Plane turbulent impinging jets. J. Hydraul. Res. 1973, 11, 29-59. [CrossRef]

17. Gutmark, E.; Wolfshtein, M.; Wygnanski, I. The plane turbulent impinging jet. J. Fluid Mech. 1978, 88, 737-756. [CrossRef]

18. Head, M.R.; Bandyopadhyay, P. New aspects of turbulent boundary-layer structure. J. Fluid Mech. 1981, 107, 297-338. [CrossRef]

19. Nakamura, I.; Tsuji, Y. Some progress in the research of the dynamical structure in wall turbulence. JSME Int. J. Ser. B Fluids Therm. Eng. 1995, 38, 335-345. [CrossRef]

20. Adrian, R.J.; Meinhart, C.D.; Tomkins, C.D. Vortex organization in the outer region of the turbulent boundary layer. J. Fluid Mech. 2000, 422, 1-54. [CrossRef]

21. Chakraborty, P.; Balachanda, S.; Adrian, R.J. On the relationships between local vortex identification schemes. J. Fluid Mech. 2005, 535, 189-214. [CrossRef]

22. Wu, Y.; Christensen, K.T. Population trends of spanwise vortices in wall turbulence. J. Fluid Mech. 2006, 568, 55-76. [CrossRef]

23. Gao, Q.; Ortiz-dueñas, C.; Longmire, E.K. Analysis of vortex populations in turbulent wall-bounded flows. J. Fluid Mech. 2011, 678, 87-123. [CrossRef]

24. Vassilicos, J.C. Dissipation in Turbulent Flows. Annu. Rev. Fluid Mech. 2015, 47, 95-114. [CrossRef]

25. Rezaeiravesh, S.; Vinuesa, R.; Liefvendahl, M.; Schlatter, P. Assessment of uncertainties in hot-wire anemometry and oil-film interferometry measurements for wall-bounded turbulent flows. Eur. J. Mech. $B$ Fluids 2018, 72, 57-73. [CrossRef]

26. Vinuesa, R.; Schlatter, P.; Malm, J.; Mavriplis, C.; Henningson, D.S. Direct numerical simulation of the flow around a wall-mounted square cylinder under various inflow conditions. J. Turbul. 2015, 16, 555-587. [CrossRef]

27. Rezaeiravesh, S.; Liefvendahl, M. Effect of grid resolution on large eddy simulation of wall-bounded turbulence. Phys. Fluids 2018, 30, 055106. [CrossRef]

28. Lee, S.; Jang, Y.; Choi, Y. Stereoscopic-PIV measurement of turbulent jets issuing from a sharp-edged circular nozzle with multiple triangular tabs. J. Mech. Sci. Technol. 2012, 26, 2765-2771. [CrossRef]

29. Cao, Z.; Wei, L.; Xie, J. Sediment-laden flow in open channels from two-phase flow viewpoint. J. Hydraul. Eng. 1995, 121, 725-735. [CrossRef]

30. Song, T.; Chiew, Y. Settling characteristics of sediments in moving Bingham fluid. J. Hydraul. Eng. 1997, 123, 812-815. [CrossRef]

31. Kiger, K.T.; Pan, C. Suspension and turbulence modification effects of solid particulates on a horizontal turbulent channel flow. J. Turbul. 2002, 3, 1-17. [CrossRef] 
32. Samanta, A.; Vinuesa, R.; Lashgari, I.; Schlatter, P.; Brandt, L. Enhanced secondary motion of the turbulent flow through a porous square duct. J. Fluid Mech. 2015, 784, 681-693. [CrossRef]

33. Rajaratnam, N.; Mazurek, K.A. An experimental study of sand deposition from sediment laden water jets. J. Hydraul. Res. 2006, 44, 560-566. [CrossRef]

34. Lyn, D.A. Turbulence characteristics of sediment-laden flows in open channels. J. Hydraul. Eng. 1992, 118, 971-988. [CrossRef]

35. Ni, J.R.; Xia, J.X. Particle fluctuation intensities in sediment-laden flows. Mech. Res. Commun. 2003, 30, 25-32. [CrossRef]

36. Guo, J.; Julien, P.Y. Turbulent velocity profiles in sediment-laden flows. J. Hydraul. Res. 2001, 39, 11-23. [CrossRef]

37. Wang, X.; Qian, N. Turbulence characteristics of sediment-laden flow. J. Hydraul. Eng. 1989, 115, 781-800.

38. Wang, Z.; Wang, X.; Ren, Y. Statistical characteristics of Bingham fluid turbulence and its fluctuation spectrum distribution. J. Hydraul. Eng. 1993, 4, 12-22. (In Chinese) [CrossRef]

39. Omid, M.H.; Nasrabadi, M.; Farhoudi, J. Suspended sediment effects on hydraulic jump characteristics. Proc. Inst. Civ. Eng. Water Manag. 2011, 164, 91-101. [CrossRef]

40. Nasrabadi, M.; Omid, M.H.; Farhoudi, J. Submerged hydraulic jump with sediment-laden flow. Int. J. Sediment Res. 2012, 27, 100-111. [CrossRef]

41. Kala, A.; Mittal, S.K.; Choudhary, M.K. Characteristics of flow meters with sediment laden flow-A review. Int. J. Eng. Res. 2015, 4, 240-243. [CrossRef]

42. Ni, J.R. Particle suspension in sediment-laden flow. Prog. Nat. Sci. 2002, 12, 481-492.

43. Surhone, L.M.; Timpledon, M.T.; Marseken, S.F. Nyquist Frequency; Betascript Publishing: Beau Bassin, Mauritius, 2010; ISBN 978-613-0-47945-9.

44. Zhang, M.; You, C.; Zhao, X. Experimental study on turbulence characteristics of high sediment-laden Flow. Water Resour. Hydropower Eng. 2016, 47, 82-88. (In Chinese) [CrossRef]

45. Lin, Z.; Hu, S.; Jiang, D.; Zhang, X. The influence of sediment concentration in muddy water on the relationship between water level and flow in rectangular groove. Res. Soil Water Conserv. 1994, 119-126. (In Chinese)

46. Davies, J.T. Turbulence Phenomena; Academic Press: New York, NY, USA; London, UK, 1972; p. 412, ISBN 9780323150934.

47. Zhou, J.G.; Shu, J.J.; Stansby, P.K. Hydraulic jump analysis for a Bingham fluid. J. Hydraul. Res. 2007, 45, 555-562. [CrossRef]

48. Wang, D. Experimental study on fluctuation pressure of sediment-laden water. Yellow River 1994, 4, 33-35. (In Chinese) 